\title{
Uncovering a Novel Stone in 27 Patients: Calcium Tartrate Tetrahydrate
}

Colin Kleinguetl, James C. Williams, Jr., John Lieske, Michel Daudon, Marcelino Rivera, Paul Jannetto, Joshua Bornhorst, Denise Rokke, Erin T. Bird, James E. Lingeman and Marawan M. El Tayeb

\section{Authors/Contacts:}

Colin Kleinguetl, MD, ScM (First author)

Affiliation: Baylor Scott \& White

Email: colin.kleinguetl@bswhealth.org

Institution Address: 2401 S. $31^{\text {st }}$ St. Temple, TX 76508

Telephone: 281-468-6003

Fax: 254-724-0218

Marawan M. El Tayeb, MD, MSc (senior author and corresponding author)

Affiliation: Baylor Scott \& White

Email: marawan.eltayeb@bswhealth.org

Institution Address: 2401 S. $31^{\text {st }}$ St. Temple, TX 76508

Telephone: 513-356-6418

Fax: 254-724-0218

James C. Williams, Jr, PhD

Affiliation: Indiana University School of Medicine, Anatomy and Cell Biology

Email: jwillia3@iupui.edu

Institution Address: 420 University Blvd, Indianapolis, IN 46202

James E. Lingeman, MD

Affiliation: Indiana University School of Medicine

Email: JLingeman@比Health.org

Institution Address: 1801 N. Senate Blvd, Indianapolis, IN 46202

Michel Daudon, PhD

Affiliation: Assistance Publique - Hôpitaux de Paris, Biochimie

Email:Michel,daudon@tnn.aphp.fr

Institution Address: Hôpital Tenon, 4 rue de la Chine, F75020 Paris

John C Lieske, MD

Affiliation: Mayo Clinic in Rochester, Minnesota

Email: lieske.john@mayo.edu

Institution Address: 200 1st St SW, Rochester, MN 55905

Erin T. Bird, MD

Affiliation: Baylor Scott \& White

Email: erin.bird@bswhealth.org

Institution Address: 2401 S. $31^{\text {st }}$ St. Temple, TX 76508

This is the author's manuscript of the article published in final edited form as:

Kleinguetl, C., El Tayeb, M. M., Williams, J. C., Lingeman, J. E., Daudon, M., Lieske, J. C., ... Rokke, D. (2019). Uncovering a Novel Stone in 27 Patients: Calcium Tartrate Tetrahydrate. Urology. https://doi.org/10.1016/j.urology.2019.01.005 
Telephone: 254-724-8277

Fax: 254-724-0218

Marcelino Rivera, MD

Affiliation: Mayo Clinic in Rochester, Minnesota

Email: rivera.marcelino@mayo.edu

Institution Address: 200 1st St SW, Rochester, MN 55905

Paul J. Jannetto, PhD

Affiliation: Mayo Clinic Department of Laboratory Medicine and Pathology

Email: Jannetto.Paul@mayo.edu

Institution Address: 200 1st St SW, Rochester, MN 55905

Joshua Bornhorst, PhD

Affiliation: Mayo Clinic Department of Laboratory Medicine and Pathology

Email: Bornhorst.Joshua@mayo.edu

Institution Address: 200 1st St SW, Rochester, MN 55905

Denise Rokke, PhD

Affiliation: Mayo Clinic Department of Laboratory Medicine and Pathology

Email: Rokke.Denise@mayo.edu

Institution Address: 200 1st St SW, Rochester, MN55905

Keywords: urolithiasis; calcium tartrate tetrahydrate; supplements; stones

\section{Disclosures/Conflict of Interest}

There are no disclosures to make and no competing financial interests exist.

\section{Abstract}

\section{Objective}

To further analyze calcium tartrate tetrahydrate stones after a recent case report described this novel stone. Prior to this, there was only one previously reported occurrence of this stone in a human. This unusual stone composition is not tested for routinely. True prevalence and possible causes of this stone are unknown.

\section{Materials/Methods}

During the previous case report, micro-CT and Fourier-transform infrared spectroscopy were used to identify a calcium tartrate tetrahydrate stone. This information was applied to urinary stones with previously unidentified compositions in the Mayo Metals laboratory database between 2010 and March 2018. Two additional stones were identified at our institution. Three patients had medical records available for analysis. 


\section{Results}

Between 2010 and March 2018, 35 calcium tartrate stones in 25 patients were identified in the Mayo database as well as two at our institution (37 stones in 27 patients). Thirty stones were pure calcium tartrate with the remainder having elements of more common stones. The average age was $46.3(+/-14.7)$ with a slightly higher incidence in females (17 vs 10).

Of the three medical records investigated, all three were males (average age 48.7), and each reported consumption of an energy supplement (Spark) routinely.

\section{Conclusions}

The true prevalence of this relatively unknown stone remains unclear and additional investigation is warranted. We believe all stone laboratories should have access to the IR spectra for calcium tartrate tetrahydrate. Attention should be payed to possible causes of this stone, particularly with relation to oral supplements, to aid with future prevention and treatment.

\section{Introduction}

Urolithiasis continues to be a significant clinical issue in both adults and children. Prevalence of stone disease has been increasing in the United States and across the globe over the last couple of decades. Overall prevalence varies between studies but may range from $7-13 \%{ }^{1}$ in North American to $15 \%$ or more ${ }^{2}$ with the most common type of urinary stones in the United States being calcium oxalate ${ }^{1}$.

We previously reported the finding of a novel kidney stone, calcium tartrate tetrahydrate in a young, otherwise healthy male ${ }^{3}$. Prior to this finding, there had only been one previously recorded calcium tartrate stone in a human being, which involved a stone in Burkina Faso. Other than noting the composition of the stone, no additional information (past medical history, social history, etc) was available ${ }^{4}$.

Calcium tartrate tetrahydrate stones have also been previously described in rat models. In these studies, there was an increased prevalence of urolithiasis noted in rats with specific purified diets that consisted of dl-choline bitartrate. Infrared spectrum of the formed stones was consistent with calcium tartrate tetrahydrate $e^{5,6}$ and was later confirmed by Le Bail et al ${ }^{7}$. In our previous case report ${ }^{3}$, our patient was noted to consume a large amount of a specific dietary supplement that contains L-carnitine (as tartrate) and choline (as bitartrate and citrate), raising the question of a possible role of dietary supplementation in formation of this stone. 
There has been ongoing debate/discussion about the effect of diet and supplements on the formation of stone. Formation is known to be asssociated with poor dietary habits, such as increased consumption of animal proteins (and decreased fruits and vegetables) and poor fluid intake that often consists of high sugar and oxalate beverages ${ }^{2,8,9}$. Certain supplements and vitamins may also play a role in increasing the rate of kidney stones. In particular, there has been significant debate with regards to vitamin $C^{10}$ and vitamin $D^{11-13}$ supplementation and stone formation. It is often recommended to avoid high doses, >500-1000mg per day, of vitamin C, while recommendations for vitamin D consumption are less clear. There currently exists a paucity of data looking at energy supplements and the formation of stones.

Here, we present findings of additional calcium tartrate tetrahydrate stones, which were uncovered by applying the Fourier-transform infrared spectrum of calcium tartrate tetrahydrate obtained from our index patient's stone. We also try to elucidate any commonality between patients diagnosed with this type of stone, specifically with regards to social history and possible dietary supplementation.

\section{Materials/Methods}

The initial finding of a calcium tartrate stone involved stone analysis that revealed a stone of unusual composition, which required the stone to be sent for additional examination at Indiana University. Micro CT imaging and Fourier-transform infrared spectroscopy (FT-IR) revealed that the majority of the stone material had $X$-ray attenuation significantly less than that expected of calcium oxalate. The infrared (IR) spectrum of the specimen did not match any known spectrum, and additional assistance was sought from the laboratory at Tenon Hospital in Paris (due to the laboratory having a more extensive database of IR spectra), which eventually revealed the identity of the stone to be calcium tartrate tetrahydrate.

With this new finding, the infrared spectrum that matched the calcium tartrate tetrahydrate stone was applied to the Mayo Metals Laboratory, specifically to kidney stones that had previously unidentified compositions over the time period of 2010-March 2018. Patient age, gender and location were available. In addition, since the index case, an additional calcium tartrate tetrahydrate stone was identified at our institution.

For at least three of the identified calcium tartrate stones (two at Baylor Scott and White and one at the Mayo Clinic), patient information was accessible for additional analysis, particularly past médical history and social history, in an effort to identify possible etiologies for this type of stone.

\section{Results}


The initially identified calcium tartrate tetrahydrate stone ${ }^{3}$ was crystalline with light coloration (Figure 1). Analysis of the micro CT demonstrated intermediate $\mathrm{x}$-ray attenuation, which was less than calcium oxalate but higher than that of cystine. Fourier-transform infrared spectroscopy (FT-IR) confirmed the identity of the stone to be calcium tartrate tetrahydrate (Figure 2).

The new spectrum for calcium tartrate tetrahydrate stone was applied to the Mayo Metals laboratory database, specifically to stones with previously unidentified spectra. A total of 35 matching stones were found in 25 different patients. Of the 35 stones, 30 of these were noted to be pure calcium tartrate. The remaining stones had compositions that included other more common stone types. One contained uric acid and calcium oxalate monohydrate, one with calcium oxalate monohydrate, one with calcium oxalate dihydrate, and one with apatite and calcium oxalate monohydrate.

During the time period from 2010 to March 2018, the first calcium tartrate stone was from 2012. There was one identified in 2013, fourteen in 2015, ten in 2016, six in 2017 and 3 in 2018 (January through March). In 2017, approximately 85000 stones were analyzed at the Mayo Metals laboratory. Of those 85000 , the composition of only 25 stones was unknown $(0.029 \%)$. When applying the FT-IR spectroscopy of calcium tartrate tetrahydrate to the unidentified stones, 6 of the stones were consistent with calcium tartrate tetrahydrate ( $24 \%$ of previously unidentified stones). This would suggest an incidence of approximately of $0.007 \%$.

From the database, the average patient age was 46.3, although the age range varied considerably, $+/-14.7$ years (oldest 81 and youngest 21 ). Seventeen of the patients were female, while eight were male. Stones were sent from across the United States with 16 from the Midwest, 2 from the Southeast, 5 from the Northeast and 2 from the West.

From our institution we added two patients (including our initial patient) with pure calcium tartrate tetrahydrate stones (both identified in 2017), bringing the total of identified stones to 37 in 27 unique patients. Both patients were male of ages 35 and 59, from the southwest (Texas).

Of the 27 patients with identified calcium tartrate stones, we were able to analyze chart information for 3 of them (two at Baylor Scott and White and one at the Mayo Clinic).

The initial calcium tartrate stone was identified in a 35-year-old male at our institution who initially presented with right sided flank pain and was diagnosed with a $2 \mathrm{~cm}$ non-obstructing stone within the right renal pelvis, with average Hounsfield units noted to be 825 . He underwent right percutaneous nephrolithotomy. He had no significant past medical history, no personal or family history of stone formation and no history of UTIs. Initial stone analysis revealed a $100 \%$ ciprofloxacin stone, which prompted additional analysis as the IR spectra was 
not consistent with ciprofloxacin and clinically did not make sense given the patient's history. The initial lab was unable to identify the stone's true composition based on the existing database. As such, the infrared (IR) spectrum was subsequently sent to the laboratory at Tenon Hospital in Paris, which identified the presence of a calcium tartrate tetrahydrate stone ${ }^{3}$. The patient had a 24-hour urinalysis performed, which was largely unremarkable, as the patient had an appropriate total volume of urine, $2.97 \mathrm{~L}$, and a pH of 6.5. Specifically, there was no evidence of hyperuricemia (555 mg/d), hypocitraturia ( $484 \mathrm{mg} / \mathrm{d})$, hyperoxaluria $(36 \mathrm{mg} / \mathrm{d})$, or hypercalciuria $(218 \mathrm{mg} / \mathrm{d})$. Only a mild elevation of urine sodium $(216 \mathrm{mEq} / \mathrm{d})$ was present. It was discovered he drank a vitamin/amino acid energy supplement, Spark by AdvoCare, every day. He reported drinking "large quantities...4 or more scoops" per day but usually averaged about 4. He had been consuming this drink consistently for a little more than a year, but he was unable to quantify the exact duration he had been consuming the product. He did continue to use the supplement from time of initial stone diagnosis, through the time of his initial 24-hour urine collection.

Our second patient is a 59-year-old male with a history of hyperlipidemia. He initially presented to the ED with right sided flank pain and CT scan revealed an $18 \mathrm{~mm}$ right renal calculus involving the right upper pole. He underwent a right PCNL that was noted to be unremarkable and patient was stone free after the case. His 24-hour urinalysis, demonstrated several abnormalities including high urine $\mathrm{pH}$ (7.4), elevated urine sodium ( $256 \mathrm{mEq} /$ day), and elevated urine phosphorous (1463 mg/day). Initial stone analysis showed stone composition of $100 \%$ calcium oxalate monohydrate (COM). However, the IR spectrum and gross appearance of the stone were not consistent with COM, and on inquiry, he reported drinking 4-5 servings of the Spark energy drink supplement every day for greater than a year (exact duration was unable to be quantified) prior to his stone episode. Due to high level of suspicion that the initial stone analysis was incorrect, the stone was retested and found to have IR spectrum consistent with $100 \%$ calcium tartrate tetrahydrate. Prior to drinking Spark, the patient had no prior history of renal colic. He continued the supplement until after his post-operative 24-hour urine analysis, a

There was a third patient identified at the Mayo Clinic. The patient is a 52-yearold male with a 20 year history of stone disease. In the past, he had passed several calcium stones and had one previous shock wave lithotripsy (ESWL) and one previous ureteroscopic extraction. Over the span of 18 months, he had noted increased frequency of stone passage, almost every month. He presented to the ED with right flank. CT showed a large burden of stone in the right renal pelvis, and he had a large branching stone obstructing in the left proximal ureter, as well as a $2 \mathrm{~cm}$ bladder stone. $\mathrm{He}$ had placement of bilateral stents followed by subsequent bilateral PCNL. Analysis of stones removed at his procedure showed stones of unknown composition on the right side and within the bladder. The left sided stone was noted to be comprised of $70 \%$ uric 
acid and $30 \%$ calcium oxalate monohydrate. Unfortunately, a 24-hour urinalysis was unavailable for this patient. Additional inquiry into the patient's social history revealed that he stopped drinking any sugary sodas as part of a weight loss program and was successful in losing approximately 45 pounds about 3 years prior to this stone episode. Over the past three years, he was drinking AdvoCare energy drinks (specifically Spark). He reported drinking two of these every day while driving a truck, 5-7 days per week.

\section{Discussion}

Until recently, calcium tartrate stones were only described in rat models with specific diets containing tartrate (dl-choline bitartrate) ${ }^{5,6,7}$ and in a single stone patient in Burkina Faso ${ }^{4}$. We were previously able to identify a calcium tartrate stone with use of micro CT and infrared spectroscopy ${ }^{3}$. Unfortunately, this spectrum is not readily available in laboratories that stones are sent to for analysis. As such, the true prevalence of this stone in the population at large is unknown. Using the previously obtained FT-IR spectrum for calcium tartrate, we were able to identify calcium tartrate stones amongst stones that previously had unknown composition. This spectrum should be available in all stone analysis laboratories to help facilitate further identification. Strong consideration should be given to re-analyzing a stone's composition if there is high level of suspicion that the initial stone analysis is incorrect, whether this be from discrepancies in the IR spectrum, gross appearance of the stone or from clinical judgment.

Analysis of the database would also suggest a very low incidence of calcium tartrate tetrahydrate stones, $\sim 0.007 \%$ based on information from 2017 . While very few stones were labeled as unidentified (25 in 2017), a significant number of those, 6 (24\%) were later re-labeled as calcium tartrate tetrahydrate with the addition of the new FT-IR spectroscopy. While this incidence is low, it may also be inaccurate. Both patients at Baylor Scott and White had reported stone compositions that were erroneous (ciprofloxacin and calcium oxalate monohydrate) and were only re-tested based due to suspicion that initial analysis was incorrect. Unfortunately, the FT-IR spectroscopy of calcium tartrate was only applied to unidentified stones, and not all stones that already had identified compositions.

Evaluation of the Mayo Metals laboratory database showed a vast range of ages for patients with this type of stone and there seemed to be a higher rate of women with this type of stone (17 female vs 10 male). However, with true prevalence unknown and a relatively small number of stones identified, it is difficult to draw accurate conclusions at this time, although the prevalence of stone disease, in general, tends to be higher in the male population.

Unfortunately, of all the identified calcium tartrate only three of the patients (all 3 male) were able to be directly questioned with regards to social history, including use of dietary supplements. A fourth patient was noted to have no mention of dietary supplements in their 
chart, but was excluded as the patient was not directly questioned. Interestingly enough, all three patients in question reported consuming the same type of supplement, Spark, for extended periods of time. This supplement contains tartrate in the forms of carnitine tartrate, L-carnitine, L-tartrate and choline (as bitartrate and citrate). A single serving (one scoop or packet) contains $500 \mathrm{mg}$ choline and $10 \mathrm{mg}$ of L-carnitine (no recommended daily consumption established by the FDA). In addition, the supplement, like many others, has significant amount of Vitamin C (180mg per serving), which means our three described patients had an intake of $360-900 \mathrm{mg}$ a day from the supplement alone. While many energy supplements contain these compounds to some degree, it is unclear to what degree they are absorbed by the GI tract or filtered by the kidneys, especially with regards to tartrate.

Although it is difficult to draw significant conclusions based on a sample size of only three patients, these cases suggest that this supplement could be a significant risk factor. We believe this warrants further investigation, and that patients should be inquired about consumption of these supplements. These types of supplements are often used for the purpose of gaining muscle mass, energy supplementation and weight loss.

\section{Conclusion}

We report additional findings of calcium tartrate tetrahydrate stones. We believe that it would be beneficial to include the IR spectra for this unique stone in additional laboratories to facilitate its future identification. Repeat stone analysis (either at the initial lab or at more specialized labs) should be considered if there is suspicion that the reported composition is incorrect. Furthermore, in three patients for which chart information was available and supplement intake was discussed, all three reported drinking the energy supplement Spark, which contains large amounts of tartrate. While this certainly does not determine causation, we believe it would be prudent to inquire about this supplement in additional stone forming patients. Additional investigation may prove beneficial in order to help prevent or reduce risk of formation of this unique stone.

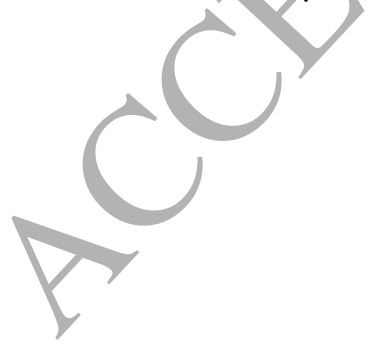




\section{Figures}

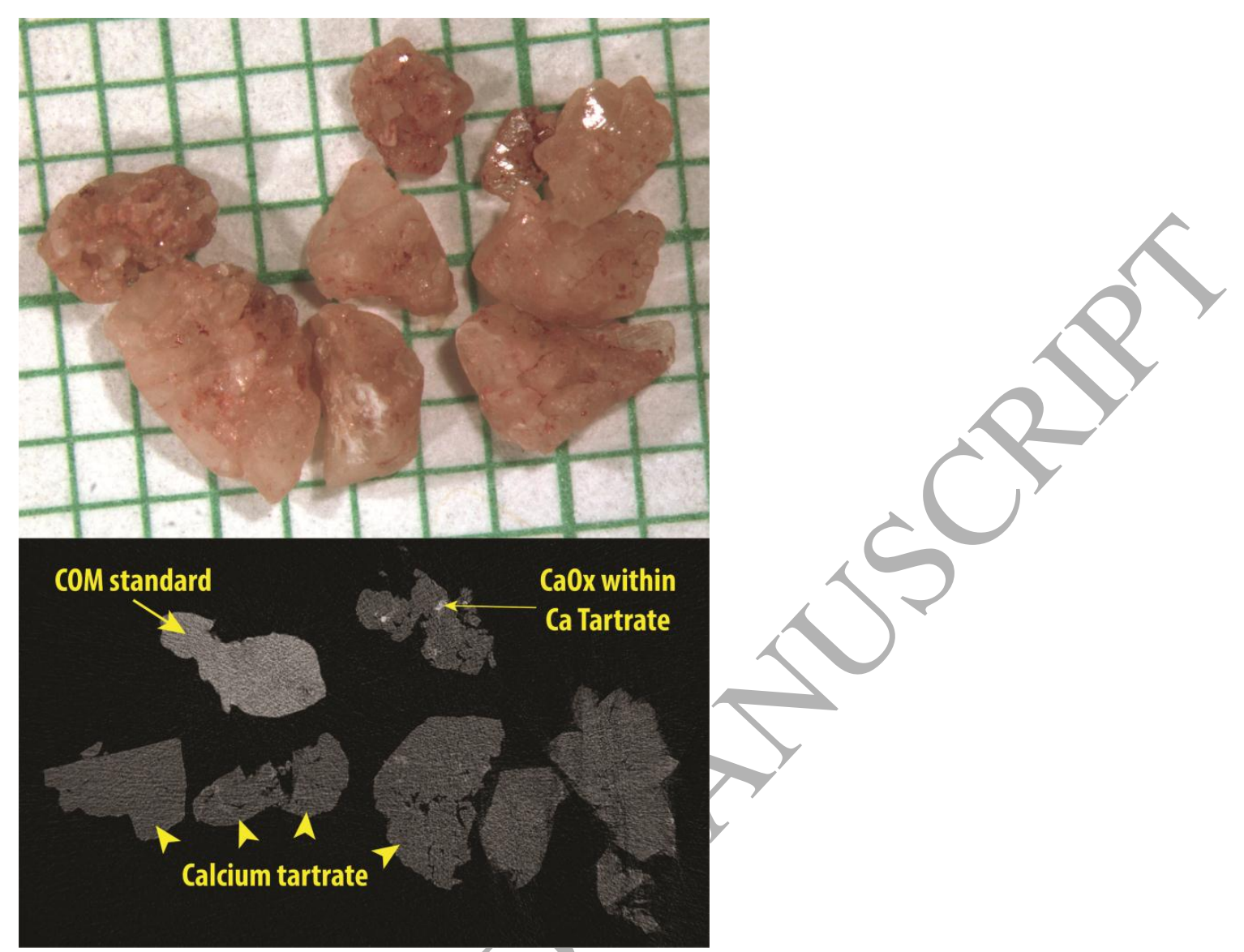

Figure 1: Photo of stone specimen on mm paper (top), with micro CT image slice (bottom). Micro CT slice includes standard specimen composed of pure calcium oxalate ( $\mathrm{CaOx}$ ) monohydrate (COM, thick arrow), and apparent $\mathrm{CaOx}$ inclusions in the specimen are indicated with thin arrow. Arrowheads indicate bulk of specimen, which was verified by infrared spectroscopy to be calcium tartrate.

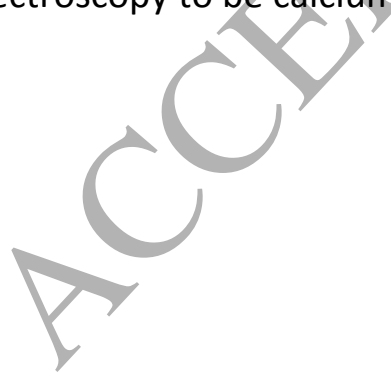




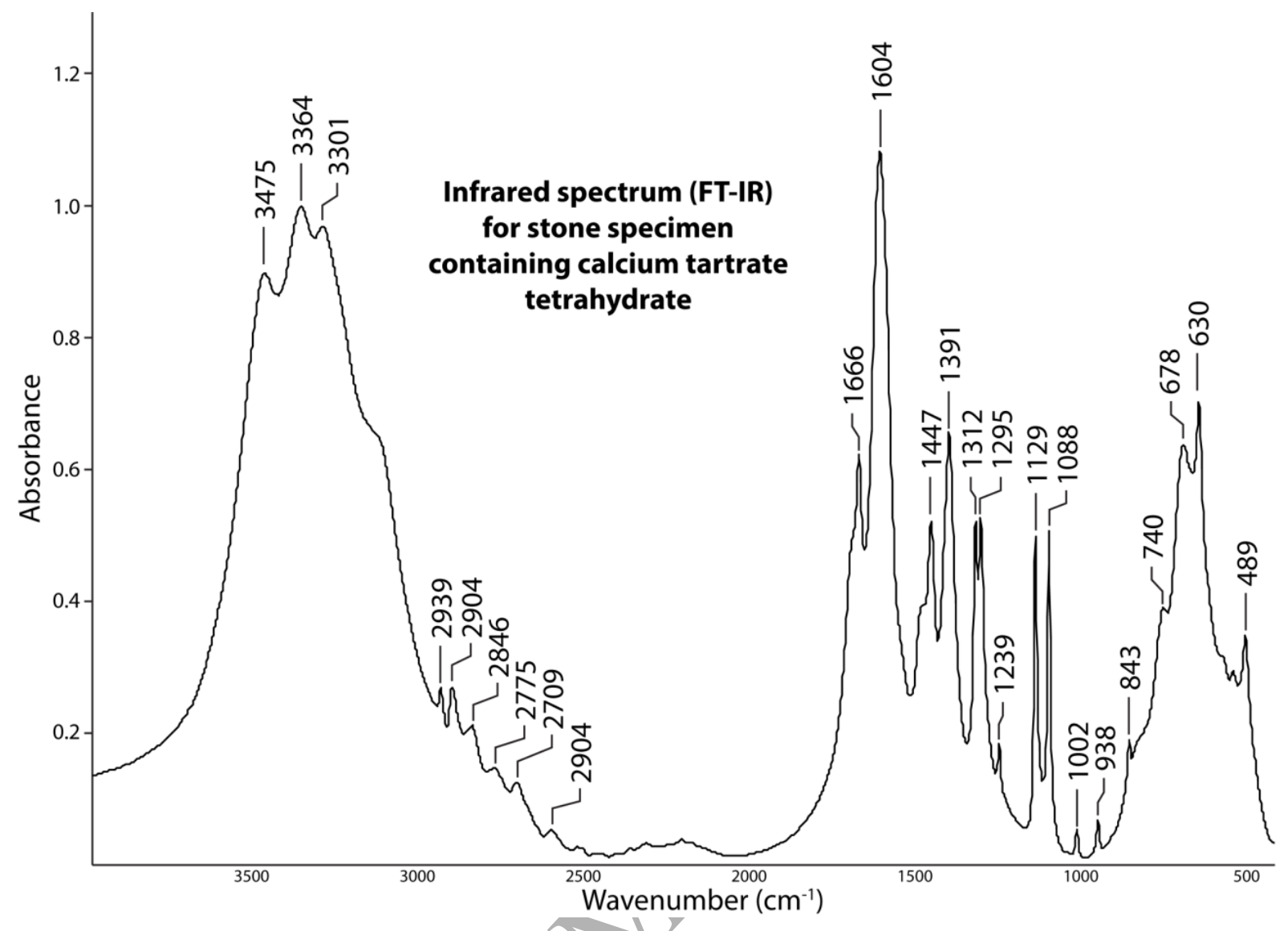

Figure 2: Fourier transform infrared (FT-IR) spectrum from the stone original stone specimen illustrating calcium tartrate tetrahydrate 


\section{References}

1) Sorokin I, Mamoulakis C, Miyazawa K, et al. Epidemiology of stone disease across the world.World J Urol. 2017.

2) Trinchieri A. Epidemiology of urolithiasis: An update. Clin Cases Miner Bone Metab. 2008;5:101-6.

3) Kleinguetl C, Williams JC Jr, Ibrahim SA, Daudon M, Bird ET, ELTayeb MM. J Endourol Case Rep. 2017 Dec 1;3(1):192-195. doi:10.1089/cren.2017.0118. eCollection 2017.

4) Dessombz, A., Kirakoya, B., Coulibaly, G. et al.: High Prevalence of Opaline Silica in Urinary Stones from Burkina Faso. Urology, 86: 1090, 2015.

5) Klurfeld, D. M. 2002. Kidney and bladder stones in rodents fed purified diets. J. Nutr. 132:3785

6) Newland MC, Reile PA, Sartin EA, et al. Urolithiasis in rats consuming a dl bitartrate form of choline in a purified diet. Comp Med. 2005 Aug;55(4):354-67.

7) Le Bail A, Bazin D, Daudon M, et al. Racemic calcium tartrate tetrahydrate [form (II)] in rat urinary stones. Acta Crystallogr B. 2009. 65:350-4.

8) Daudon, M., Donsimoni, R., Hennequin, C. et al.: Sex and age-related composition of 10617 calculi analyzed by infrared-spectroscopy. Urol Res, 23: 319, 1995. 
9) Sakhaee K, Maalouf NM, Sinnott B. Clinical review. Kidney stones:

Pathogenesis, diagnosis, and management. J Clin Endocrinol Metab. 2012;97:1847-60.

10) Baxmann AC, Mendonca CD, Heilberg IP. Effect of vitamin C supplements on urinary oxalate and $\mathrm{pH}$ in calcium stone-forming patients. Kidney Int 2003;63: 1066-71

11) Hesswani C, Noureldin YA, Elkoushy MA, Andonian S. Combined vitamin D and calcium supplementation in vitamin D inadequate patients with urolithiasis: impact on hypercalciuria and de novo stone formation. Can Urol Assoc J. 2015. $9(11-12): 403-408$

12) Malihi Z, Wu Z, Stewart AW, Lawes CM, Scragg R. Hypercalcemia, hypercalciuria, and kidney stones in long-term studies of vitamin D supplementation: a systematic review and meta-analysis. Am J Clin Nutr. 2016. 104:1039-1051

13) Hesswani C, Noureldin YA, Elkoushy MA, Andonian S. Combined vitamin D and calcium supplementation in vitamin D inadequate patients with urolithiasis: impact on hypercalciuria and de novo stone formation. Can Urol Assoc J. 2015 $9(11-12): 403-408$. 